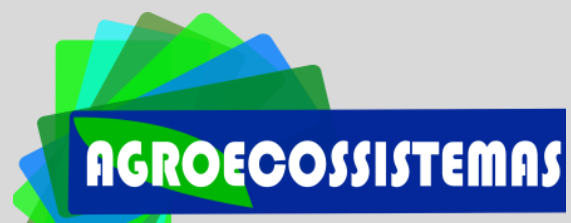

Núcleo de Meio Ambiente Universidade Federal do Pará Rua Augusto Corrêa, 01, Guamá Belém, Pará, Brasil

https://periodicos.ufpa.br/index.php/agroecossistemas

Jonilson Santos de Carvalho Universidade Estadual do Sudoeste da Bahia jonilsonif@gmail.com

Abel Rebouças São José Universidade Estadual do Sudoeste da Bahia abeljose3@gmail.com

Alessandro de Magalhães Arantes Instituto Federal de Educação, Ciência e Tecnologia Baiano arantes2005@yahoo.com.br

Alcebíades Rebouças São José Universidade Estadual do Sudoeste da Bahia alreboucas@gmail.com

Maira de Araújo Kurfis Instituto Federal de Educação, Ciência e Tecnologia Baiano maira_kurfis@hotmail.com

Recebido em: 2019-10-04 Avaliado em: 2020-09-10 Aceito em: 2021-02-10

\section{SOBREVIVÊNCIA DE ESPÉCIES DE MARACUJAZEIRO COM OU SEM EXPOSIÇÃO PARCIAL DAS RAÍZES, EM ÁREA COM HISTÓRICO DE Fusarium solani}

RESUMO: A passicultura destaca-se como uma importante atividade econômica em várias regiões do Brasil, sobretudo no Nordeste, que detém mais de 60\% da produção nacional. Ainda assim, o sistema de produção da cultura do maracujá é carente em tecnologias, sendo este um dos motivos do aumento da incidência de doenças e baixa produtividade da cultura. Entre as doenças importantes para o maracujazeiro no Brasil, destaca-se a podridão do colo e raiz, causada pelo Fusarium solani (Mart.) Sacc., principalmente por causar a morte das plantas infectadas e ainda não existir um manejo curativo. Portanto, o objetivo deste trabalho foi avaliar a sobrevivência de três espécies de maracujazeiro: Passiflora edulis Sims, Passiflora cincinnata Mast e Passiflora foetida L., submetidas ao sistema de plantio com e sem exposição parcial das raízes, avaliadas em dez

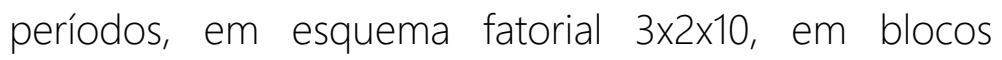
casualizados. A incidência de F. solani foi realizada por meio de levantamento semanal do número de plantas mortas. O experimento foi conduzido no período de janeiro a junho de 2019, em uma área de 0,3 ha com histórico de F. solani, localizada no Instituto Federal Baiano campus Guanambi. Verificou-se que a exposição parcial do sistema radicular reduz o percentual de plantas mortas por F. solani, e, dentre as espécies avaliadas, destaca-se $\circ P$. foetida como altamente resistente ao $F$. solani. Portanto, o uso de estratégias de manejo integradas é uma alternativa viável aos passicultores para mitigar os danos causados pela podridão do colo e raiz.

PALAVRAS-CHAVE: Biocontrole, Fitossanidade, Passiflora. 


\section{SURVIVAL OF PASSION FRUIT SPECIES WITH OR WITHOUT PARTIAL ROOT EXPOSURE IN AN AREA WITH HISTORY DISEASE OF Fusarium solani}

ABSTRACT: The passion fruit crop stands out as an important economic activity in several regions of Brazil, especially in the Northeast, which holds more than $60 \%$ of the national production. Nevertheless, the passion fruit production system is lacking in technologies, this is one of the reasons for the increase in disease incidence and low productivity. There are important passion fruit diseases in Brazil, among which root and collar rot caused by Fusarium solani (Mart.) Sacc. mainly because it causes the death of infected plants and there is still no curative management. Therefore, this work aimed to evaluate the survival of three species of passion fruit: Passiflora edulis Sims, Passiflora cincinnata Mast and Passiflora foetida L., submitted to the system of planting with and without partial exposure of the roots, evaluated over ten periods, in a factorial scheme $3 \times 2 \times 10$, in randomized block. The incidence of $F$. solani was rated on a weekly basis by the number of dead plants. The experiment was conducted from January to June 2019, in an area of 0.3 ha with a history of F. solani incidence, located at the Instituto Federal Baiano campus Guanambi. It was verified that the partial exposure of the root system reduces the percentage of dead plants by F. solani and, among the evaluated species, stands the $P$. foetida as highly resistant to $F$. solani. Therefore, using integrated management strategies is a viable alternative for farmers to mitigate the damage caused by passion fruit root and collar rot.

KEY-WORDS: Biocontrol, phytosanitary, Passiflora.

\section{SUPERVIVENCIA DE GENOTIPOS DE MARACUYÁ CON Y SIN EXPOSICIÓN PARCIAL DE RAÍCES EN UN ÁREA CON ANTECEDENTES DE Fusarium solani}

RESUMEN: La pasicultura se destaca como una actividad económica importante em varias regiones de Brasil, especialmente en el Noreste, que posee más del 60\% de la producción nacional. Aun así, el sistema de producción de cultivos de maracuyá carece de tecnologías, que es una de las razones de la mayor incidencia de enfermedades y la baja productividad del cultivo. Entre las enfermedades importantes para la fruta de la pasión en Brasil, se destaca la podredumbre del cuello y raíces, causada por Fusarium solani (Mart.) Sacc., principalmente porque causa la muerte de plantas infectadas y no hay un tratamiento curativo. El objetivo de este trabajo fue evaluar la supervivencia de tres especies de maracuyá: Passiflora edulis Sims, Passiflora cincinnata Mast e Passiflora foetida L., sometidas al sistema de plantación con y sin exposición parcial a la raíz, evaluado durante diez períodos, en un esquema factorial $3 \times 2 \times 10$, en bloques al azar. La incidencia de F. solani se realizó mediante una 
evaluación semanal del número de plantas muertas. El experimento se realizó de enero a junio de 2019, en un área de 0.3 ha con historia de F. solani, ubicado en el Instituto Federal Baiano campus Guanambi. La exposición parcial del sistema radicular redujo el porcentaje de plantas muertas por F. solani, y entre las especies evaluadas, $P$. foetida se destaca como altamente resistente a F. solani. Por tanto, el uso de estrategias de manejo integradas es una alternativa viable para que los agricultores mitiguen el daño causado por la podredumbre del cuello y raíces

PALABRAS CLAVE: Biocontrole, sanidade vegetal, passiflora.

\section{INTRODUÇÃO}

O maracujá-amarelo (Passiflora edulis Sims) destaca-se entre as espécies de passifloráceas cultivadas e está presente em mais de 90\% dos pomares de maracujazeiro no território nacional (MELETTI, 2011; JESUS et al., 2017). Essa preferência é em função de um conjunto de características, tais como maior vigor, maior produtividade, maior acidez e melhor rendimento de suco (FERREIRA et al., 2016). Entretanto, essa espécie enfrenta diversos problemas fitossanitários, ao ponto de ter o tempo de cultivo reduzido de perene para anual, o que a torna inviável em algumas regiões (KUDO et al., 2012).

Dentre as principais doenças que reduzem o potencial produtivo do maracujazeiro, pode-se destacar a podridão do colo e raiz, causada pelo fungo Fusarium solani (FISCHER et al., 2005a; BUENO et al., 2010). Devido à inexistência de medidas efetivas de manejo dessa doença, ocorre redução da longevidade do pomar, menor produtividade e o aumento no custo de produção, o que resulta no abandono de área (SÃO JOSÉ et al., 2011).

Diante do risco de redução das áreas cultivadas, diversas estratégias têm sido testadas para ampliar a viabilidade do cultivo, dentre as quais destacam-se o uso de material genético resistente (CAVICHIOLI et al., 2011; PREISIGKE et al., 2017) e a utilização de biofungicidas (BOMFIM et al., 2010; SILVA et al., 2014). Além disso, 
estratégias como o plantio com exposição parcial do sistema radicular, provenientes do conhecimento empírico dos passicultores para mitigar os danos causados por alguns fungos de solo, necessitam de comprovação científica.

Das estratégias promissoras, o uso de material genético resistente pode ser um dos métodos eficientes no manejo das doenças causadas por patógenos de solo, no entanto ainda não existe registro de cultivares comerciais de maracujazeiro resistentes à podridão do colo e raiz (SANTOS FILHO et al., 2017). A avaliação e seleção de espécies com melhor desempenho, com potencial para serem usados como portaenxerto, também são vistas como uma das alternativas viáveis (SILVA et al., 2017).

Nesse contexto, o Passiflora cincinnata é uma das espécies consideradas potencialmente importantes para uso como portaenxerto, haja vista que apresenta moderada tolerância aos fungos patogênicos do solo, comparado ao $P$. edulis; assim como o $P$. foetida, que é um material genético ainda pouco explorado, mas com potencial para ser usado como porta enxerto e no melhoramento genético (PREISIGKE et al., 2017).

Outra opção para combater a doença seria a mudança do ambiente em que se dá o ótimo desenvolvimento do patógeno (MARCHIONATTO, 1949). A partir disso e com base na experiência de alguns passicultores, que têm utilizado a exposição parcial do sistema radicular como um método de manejo ao F. solani, infere-se que esta possa ser uma prática viável, fundamentada no princípio da regulação.

Desse modo, é pertinente avaliar se o manejo do sistema de plantio, com ou sem exposição parcial das raízes, é uma alternativa viável para a redução da mortalidade das plantas em área com histórico de F. solani. Para isso, objetivou-se avaliar a sobrevivência de três espécies de maracujazeiro ( $P$. edulis, P. cincinnata e P. foetida), 
submetidas ao sistema de plantio com ou sem exposição parcial do sistema radicular, em área com histórico do F. solani.

\section{MATERIAL E MÉTODOS}

DESCRIÇÃO DAS CONDIÇÕES EXPERIMENTAIS

O experimento foi conduzido no período de janeiro a junho de 2019, em uma área de 0,3 ha localizada no Instituto Federal Baiano campus

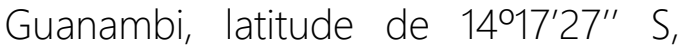
longitude de $42^{\circ} 46^{\prime} 53^{\prime \prime}$ W, altitude de 537 m, em um latossolo VermelhoAmarelo distrófico típico, A fraco, textura média, fase caatinga hipoxerófila.

A precipitação média anual é de 680 mm, e a temperatura média anual, de $26^{\circ} \mathrm{C}$. A região localiza-se no Grande Domínio Morfoclimático da Caatinga. Segundo a Classificação climática de Koppen, a região tem clima quente (Aw), com estação seca bem definida, coincidindo com o inverno. $O$ período chuvoso varia de outubro a março e apresenta pelo menos um mês com precipitação inferior a $60 \mathrm{~mm}$. A temperatura do mês mais frio é superior a $18^{\circ} \mathrm{C}$, e a amplitude térmica das médias mensais mantém-se abaixo de $5^{\circ} \mathrm{C}$ (BRASIL, 1982).

As variáveis meteorológicas registradas durante 0 período experimental, temperaturas máximas e mínimas, precipitação, umidade relativa e velocidade média do vento e rajadas foram coletadas da estação meteorológica automática (Modelo Vantage Pro Integrated Sensor, fabricado pela Davis Instruments, Wayward, Califórnia, USA), instalada próxima à área de implantação do experimento, quando se verificaram temperaturas máximas próximas de 40 ${ }^{\circ} \mathrm{C}$, alguns eventos de chuvas em fevereiro, março e abril, ventos moderados a fortes, com rajadas de até $60 \mathrm{~km} \mathrm{~h}^{-1}$ (Figura 1).

As informações do solo foram obtidas a partir de 15 amostras simples do solo destinado à implantação da área experimental, em profundidades de 0-20 cm, para formar uma amostra composta, que, posteriormente, foram 
enviadas ao Laboratório de Solos da Universidade Estadual do Sudoeste da Bahia - UESB, campus Vitória da
Conquista, para determinação das propriedades

físico-

Figura 1. Temperatura máxima, mínima e média $\left({ }^{\circ} \mathrm{C}\right)(\mathrm{A})$; precipitação $(\mathrm{mm})$ e umidade relativa do ar (\%) (B); velocidade média do vento $\left(\mathrm{m} \mathrm{s}^{-1}\right)$ e rajadas de vento $\left(\mathrm{km} \mathrm{h}^{-1}\right)$ (C), registrados na estação meteorológica localizada no IF Baiano, campus Guanambi, no período de janeiro a junho de 2019.
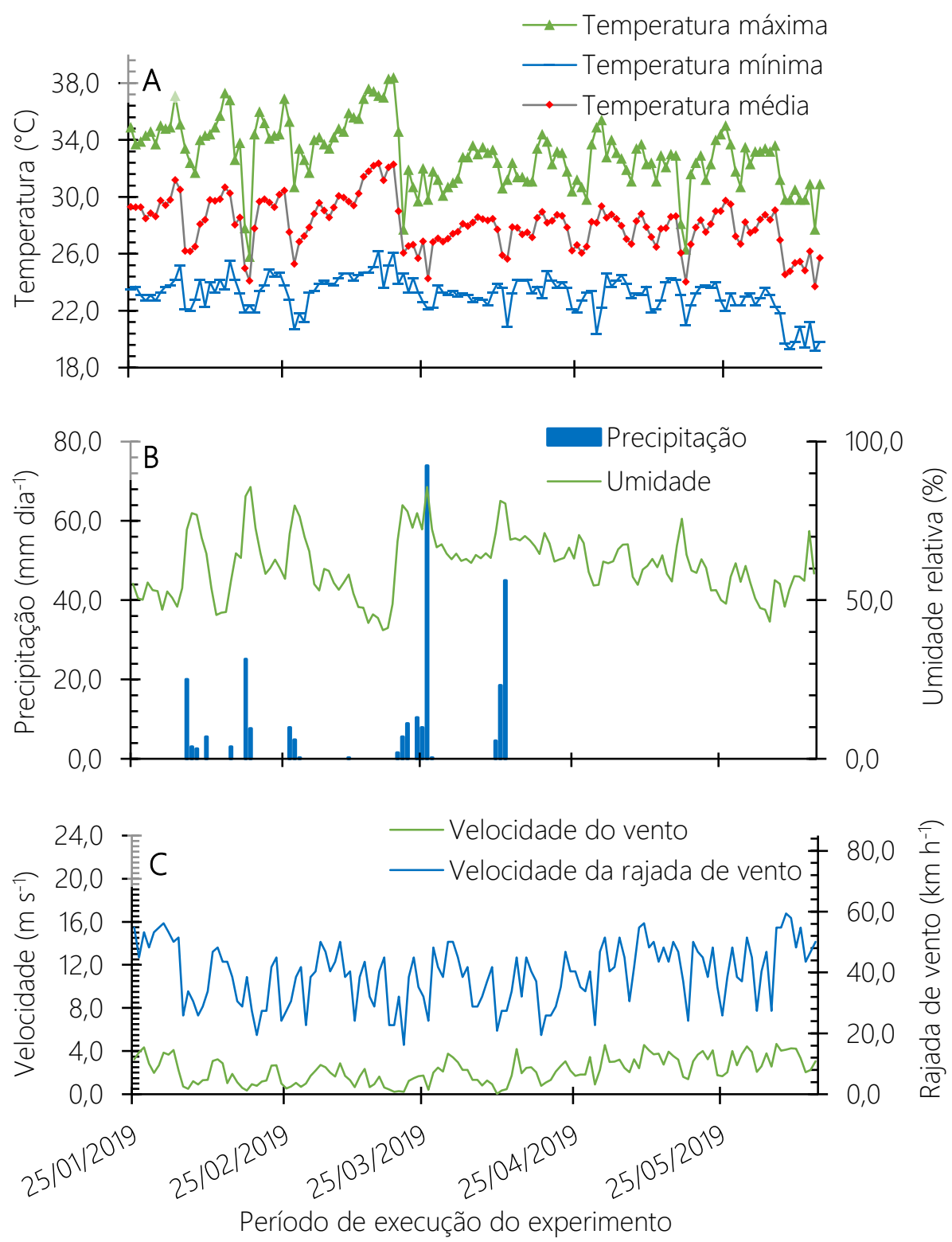

Fonte: Elaborado pelo autor. 
Tabela 1. Caracterização físico-química do solo da área experimental. Guanambi - BA, 2018

\begin{tabular}{|c|c|c|}
\hline \multirow{2}{*}{ Parâmetros } & \multirow{2}{*}{ Unidade } & \multirow{2}{*}{ Profundidade } \\
\hline & & \\
\hline $\mathrm{pH}\left(\mathrm{H}_{2} \mathrm{O}\right)$ & -- & 7,50 \\
\hline$P$ & $\mathrm{mg} \mathrm{dm}{ }^{-3}$ & 49,00 \\
\hline $\mathrm{K}^{+}$ & $\mathrm{cmol}_{\mathrm{c}} \mathrm{dm}^{-3}$ & 0,36 \\
\hline $\mathrm{Ca}^{2+}$ & $\mathrm{cmol}_{\mathrm{c}} \mathrm{dm}^{-3}$ & 3,20 \\
\hline $\mathrm{Mg}^{2+}$ & $\mathrm{cmol}_{\mathrm{c}} \mathrm{dm}^{-3}$ & 2,30 \\
\hline $\mathrm{Al}^{3+}$ & $\mathrm{cmol}_{\mathrm{c}} \mathrm{dm}^{-3}$ & 0,00 \\
\hline $\mathrm{H}^{+}$ & $\mathrm{cmol}_{\mathrm{c}} \mathrm{dm}^{-3}$ & 0,90 \\
\hline $\mathrm{Na}^{+}$ & $\mathrm{cmol}_{\mathrm{c}} \mathrm{dm}^{-3}$ & 0,13 \\
\hline$S . B^{1}$ & $\mathrm{cmol}_{\mathrm{c}} \mathrm{dm}^{-3}$ & 6,00 \\
\hline$t^{2}$ & $\mathrm{cmol}_{\mathrm{c}} \mathrm{dm}^{-3}$ & 6,00 \\
\hline$T^{3}$ & $\mathrm{cmol}_{\mathrm{c}} \mathrm{dm}^{-3}$ & 6,90 \\
\hline$V^{4}$ & $\%$ & 87,00 \\
\hline$m^{5}$ & $\%$ & 0,00 \\
\hline $\mathrm{PST}^{6}$ & $\%$ & 2,00 \\
\hline M.O. ${ }^{7}$ & $\mathrm{~g} \mathrm{dm}^{-3}$ & 7,00 \\
\hline
\end{tabular}

Classe textural Franco arenoso

${ }^{1}$ soma de bases; ${ }^{2}$ capacidade de troca catiônica efetiva, CTC efetiva; ${ }^{3} \mathrm{CTC}$ a pH 7,0; ${ }^{4}$ saturação por bases; ${ }^{5}$ saturação por alumínio; ${ }^{6}$ porcentagem de Sódio Trocável; ${ }^{7}$ matéria orgânica do solo. FONTE: Laboratório de solos da UESB, 2017.

\section{CONDUÇÃO DO EXPERIMENTO}

As plantas foram irrigadas por gotejamento com vazão de $8 \mathrm{~L} \mathrm{~h}^{-1}$ e um gotejador por planta. As irrigações foram realizadas com base na evapotranspiração de referência (ETo) determinada pelo método de PenmanMonteith, por meio de elementos meteorológicos coletados diariamente numa estação meteorológica instalada próximo à área. Os coeficientes de cultivo para determinação da ETC foram definidos em função das fases fenológicas.

Foram plantadas mudas de capim Napier (Pennisetum purpureum Schum.) para servirem como quebraventos em torno da área experimental, nas laterais de predomínio dos ventos. Os tratos culturais recomendados ao maracujazeiro foram adotados conforme recomendações técnicas 
desenvolvidas para a cultura. A fertilização foi realizada com base em análise de solo e recomendações de adubação para o maracujazeiro (BORGES e SOUZA, 2010).

A partir de 15 dias após o transplantio, foi iniciada a condução das plantas, com eliminação manual de todos os brotos laterais, deixando-se apenas o caule principal, que foi conduzido em haste única por um barbante de algodão, amarrado em uma pequena estaca fixada ao solo, até - fio do arame da espaldeira. Posteriormente, assim que as plantas atingiram 1,8 m, foram guiadas para um lado e conduzidas pelo arrame. Os ramos laterais que formaram a cortina foram podados a $20 \mathrm{~cm}$ do solo (FIGUEIREDO et al., 2015).

As entrelinhas de cultivo receberam roçagens periódicas, por meio de roçadeira acoplada à tomada de força do trator, e, nas linhas, utilizou-se roçadeira costal; a biomassa produzida foi adicionada à linha de cultivo para formar uma camada de cobertura morta que serviu de barreira mecânica para as plantas espontâneas, uma vez que se evita o uso de capina mecânica por meio da enxada em áreas afetadas por F. solani. As pragas que ocorreram ao longo do experimento foram controladas de acordo com as recomendações disponíveis na base de dados AGROFIT (MAPA, 2018).

\section{TRATAMENTO E DELINEAMENTO EXPERIMENTAL}

O ensaio foi instalado em blocos casualizados, em esquema fatorial

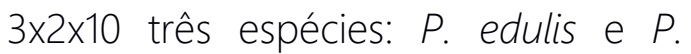
cincinnata, obtidas da seleção de sementes da região de Guanambi, e $P$. foetida, oriunda de Mossoró - RN, submetidas ao sistema de plantio convencional, sem exposição do sistema radicular, e com sistema radicular parcialmente exposto, avaliados em dez épocas, em uma área naturalmente infestada com $F$. solani. Utilizaram-se três repetições e sete plantas úteis por parcela, avaliadas a cada 7 dias.

O método de plantio com exposição parcial do sistema radicular foi aplicado 
por meio da elevação das covas a 15 cm do nível do solo, e, aos 60 dias após

o transplantio (DAT), realizou-se a aplicação de um jato de água junto ao colo da planta de modo que se

expusessem os primeiros seis centímetros do sistema radicular (Figura 2).

Figura 2. Covas elevadas a $15 \mathrm{~cm}$ do nível do solo (A e B); jato de água aplicado para exposição parcial do sistema radicular (C); sistema radicular exposto (D). Guanambi BA, 2019.
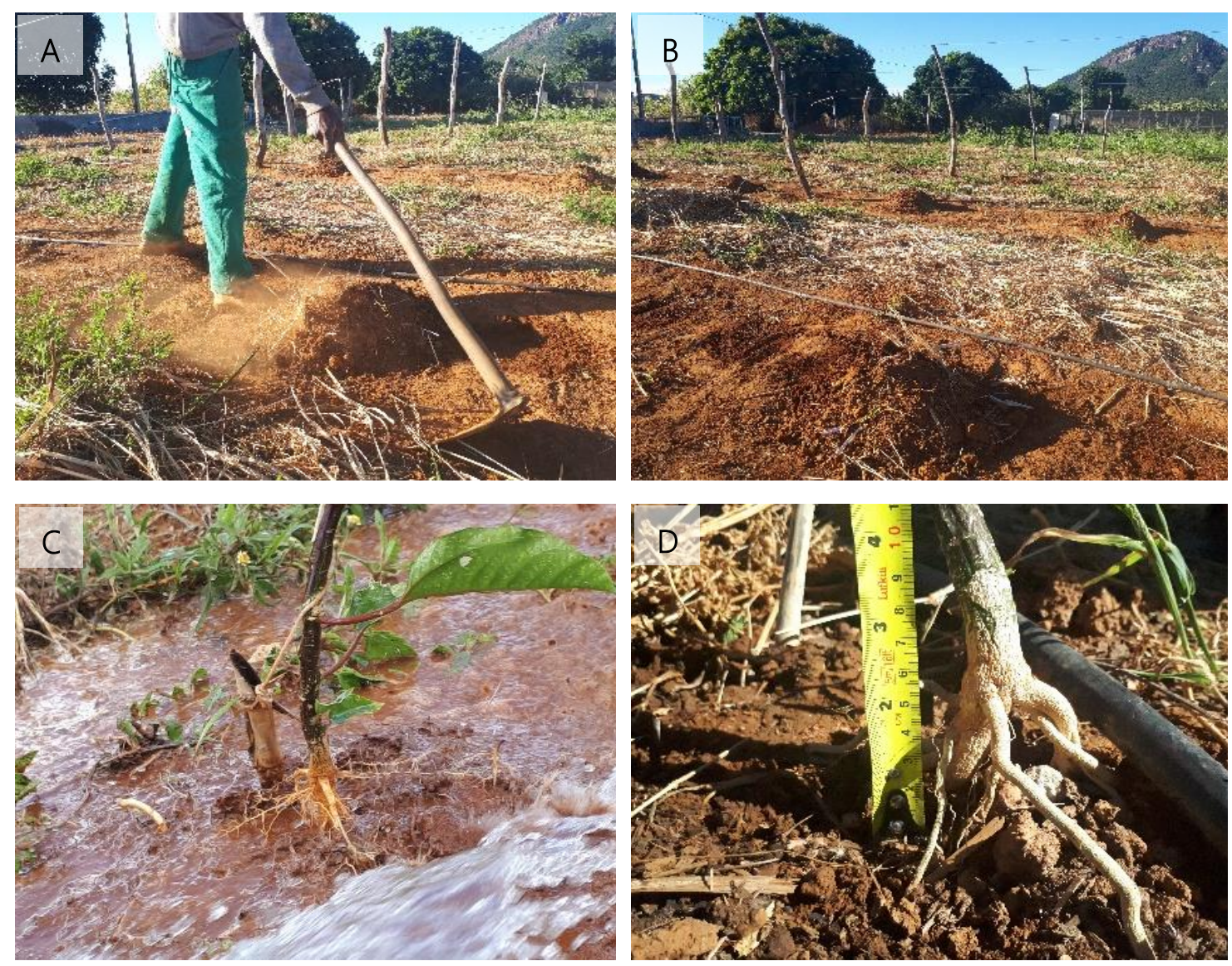

Fonte: Elaborado pelo autor. 
AVALIAÇÃO DE INCIDÊNCIA DA DOENÇA

A incidência de sintomas associados a F. solani foi realizada por meio de levantamento semanal do número de plantas mortas. Foi considerada como morta toda planta que iniciou $\mathrm{O}$ processo de murcha nas folhas, uma vez que este é um processo irreversível (JESUS et al., 2016). Além da presença de murcha, os sintomas característicos presentes no colo e sistema radicular foram observados.

As plantas infectadas foram facilmente destacadas do solo, pois grande parte do sistema radicular já havia sido necrosado e as poucas raízes remanescentes tornaram-se curtas, de coloração escurecida e quebradiças, devido à atuação do fungo (Figura 3A). A partir desse levantamento, calculou-se o percentual de sobrevivência ao longo do tempo e a longevidade das plantas.
Para a confirmação do agente etiológico na área, realizou-se no Laboratório do IF Baiano campus Guanambi o isolamento do fungo por meio do material retirado do colo e raiz de plantas com os sintomas da doença. Após isolado, uma amostra foi encaminhada à Embrapa Mandioca e Fruticultura para a confirmação da presença do patógeno na área (Figura 3B e 3C).

\section{ANÁLISE ESTATIÍSTICA}

Os dados foram submetidos à análise de variância, e as interações foram desdobradas quando se obteve significância. As médias das variáveis foram comparadas pelo teste $\mathrm{F}$ e de Tukey para os fatores sistema de plantio e espécies, respectivamente. $\bigcirc$ percentual de sobrevivência ao longo do tempo foi analisado por meio de Regressão Polinomial, a $5 \%$ de significância. 
Figura 3. Planta com sintoma da podridão do colo e raiz (A); F. solani isolado da área, frente (B) e verso (C) da placa de Petri. Guanambi - BA, 2019

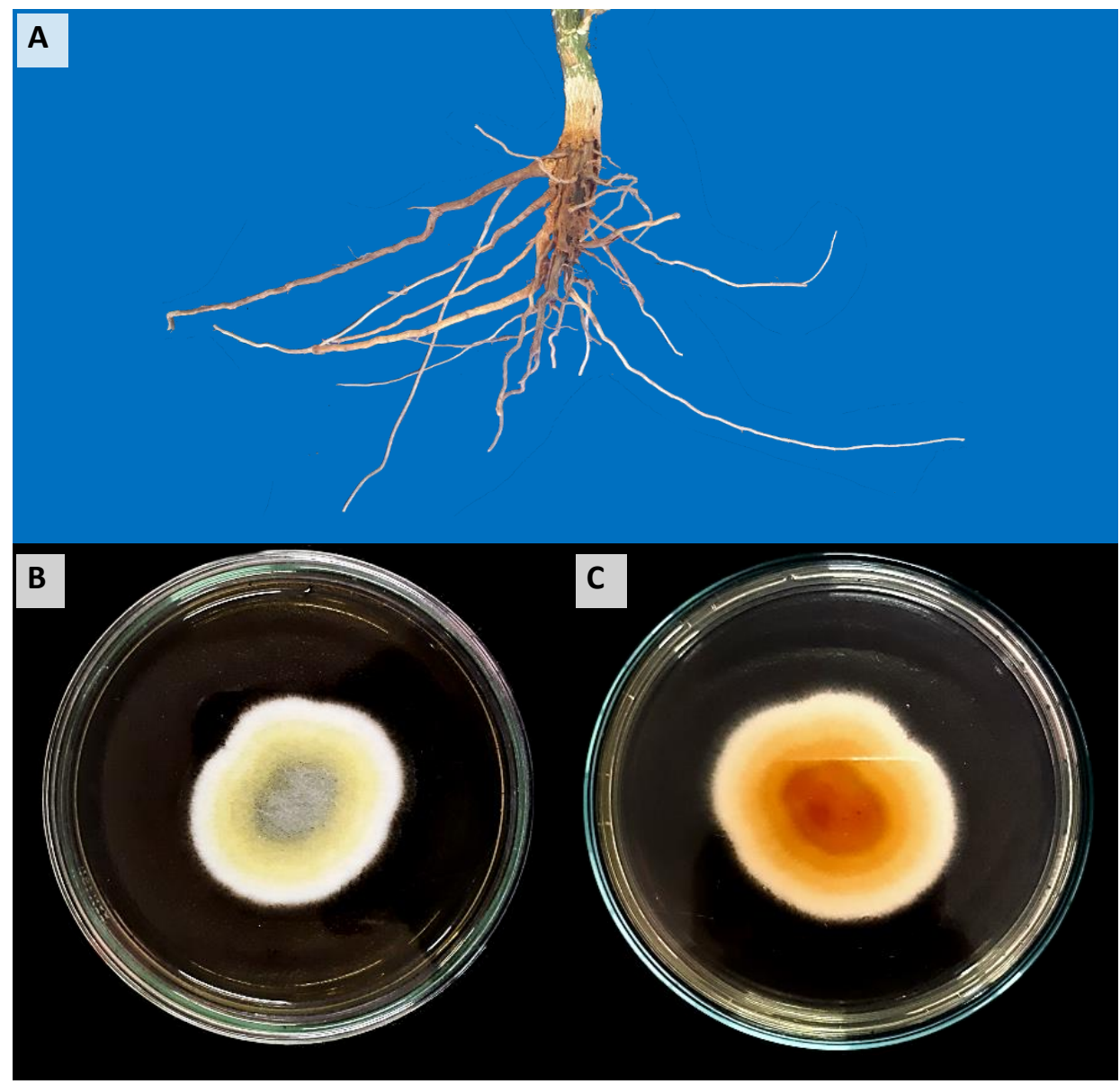

Fonte: Elaborado pelo autor.

\section{RESULTADOS E DISCUSSÃO}

As espécies de maracujazeiro, $P$. edulis, P. cincinnata e P. foetida, apresentaram interação significativa com o período de avaliação $(p<0,01)$, assim como houve interação significativa entre as espécies e o manejo aplicado ao sistema de plantio com ou sem exposição parcial do sistema radicular $(p<0,01)$, para o percentual de sobrevivência, avaliado até aos 140 DAT (Tabela 2). Contudo, não foi observado interação tripla entre os fatores analisados. 
Tabela 2. Resumo da análise de variância com os respectivos quadrados médios para percentual de sobrevivência até os 140 dias após o transplantio (DAT) de diferentes espécies de maracujazeiro em área com histórico de F. solani, submetidos ao sistema de plantio com e sem exposição parcial do sistema radicular. Guanambi, Bahia, 2019.

\begin{tabular}{|c|c|c|}
\hline \multirow{2}{*}{ FV } & \multirow{2}{*}{$\mathrm{GL}$} & Quadrados médios \\
\hline & & Percentual de sobrevivência \\
\hline Espécie (E) & 2 & $5341,26^{\star \star}$ \\
\hline Manejo (M) & 1 & $1160,99 * *$ \\
\hline DAT & 9 & $722,60^{\star \star}$ \\
\hline$E \times M$ & 2 & $280,04^{\star *}$ \\
\hline EXDAT & 18 & $371,50^{* *}$ \\
\hline$M \times D A T$ & 9 & $42,32^{\mathrm{NS}}$ \\
\hline$E \times M \times D A T$ & 18 & $34,39^{N S}$ \\
\hline Bloco & 2 & $280,04^{\star \star}$ \\
\hline Resíduo & 118 & \\
\hline
\end{tabular}

O P. edulis apresentou o primeiro registro de mortalidade aos 15 DAT, com percentual de sobrevivência decrescente em um modelo linear, 4,3\% a cada 15 dias e sobrevivência média de 54,76\% aos 140 DAT (Figura 4). Já o $P$. cincinnata só apresentou registro de mortalidade aos 76 DAT (Figura 4), sendo considerado uma espécie de resistência moderada (SILVA et al., 2013; PREISIGKE et al., 2017). Para o P. foetida, entretanto, foi registrada uma única morte aos 91 DAT (Figura 4), por isso, foi avaliado como altamente resistente
(SILVA et al., 2013; PREISIGKE et al., 2015a; CARVALHO et al., 2021), com potencial para ser utilizado como portaenxerto ao $P$. edulis.

A exposição parcial do sistema radicular reduziu o percentual de mortalidade para as plantas de $P$. edulis, com uma taxa de sobrevivência de $23,8 \%$ superior em relação às plantas sem a exposição parcial do sistema radicular, aos 140 DAT. As espécies $P$. foetida e $P$. cincinnata foram superiores ao $P$. edulis tanto no sistema de plantio com exposição do 
sistema radicular quanto na ausência do manejo (Figura 5). Ambos apresentaram percentual de sobrevivência superior aos 90\%, com destaque para o P. foetida, com 100\% de sobrevivência quando no sistema de plantio com exposição parcial das raízes (Figura 5).

Figura 4. Percentual de sobrevivência média de diferentes espécies de maracujazeiro, em área com histórico de F. solani, independente do manejo do sistema radicular, no período de janeiro a junho de 2019, em Guanambi, Bahia, 2019.

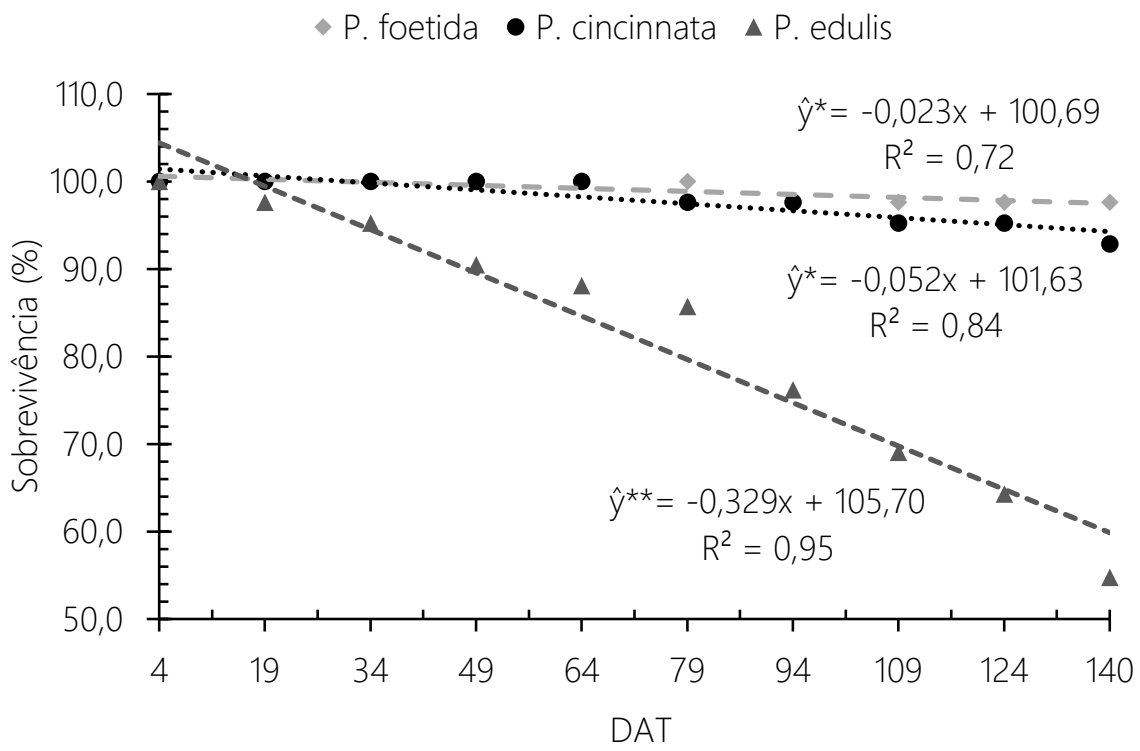

*significativo a 5\% e **significativo a $1 \%$ de probabilidade pela Análise de Variância de Regressão. Fonte: Elaborado pelo autor.

Alguns autores, utilizando como parâmetro a análise de sobrevivência, observaram que o maracujazeiro amarelo apresentou baixo percentual de sobrevivência em relação às demais espécies (LARANJEIRA et al., 2005;
PREISIGKE et al., 2017), verificado também em trabalho com vários híbridos e cultivares comerciais (SILVA et al., 2017), o que reforça a necessidade de alternativas tecnológicas para o cultivo do P. edulis. 
Figura 5. Percentual de sobrevivência média de diferentes espécies de maracujazeiro, em área com histórico de F. solani, submetidas ao sistema de plantio com exposição do sistema radicular (linhas seccionadas e marcadores preenchidos) e sem exposição do sistema radicular (linhas sólidas e marcadores sem preenchimento) no período de janeiro a junho de 2019, em Guanambi, Bahia, 2019.

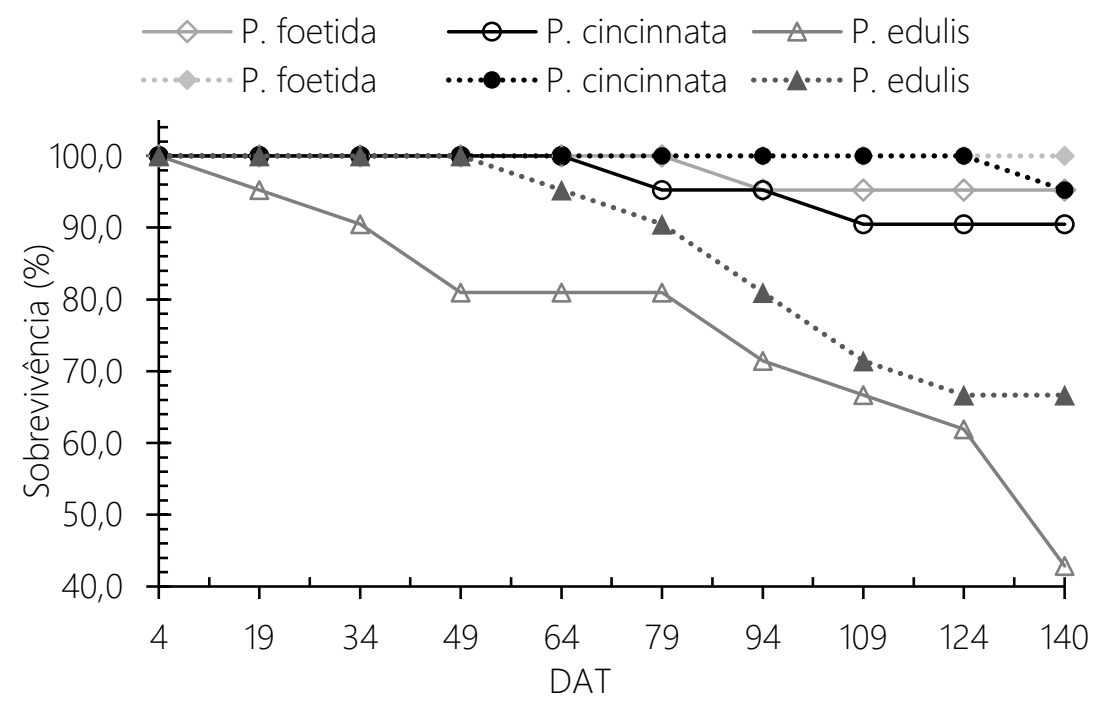

Fonte: Elaborado pelo autor.

A espécie $P$. cincinnata mostrou-se razoavelmente tolerante ao F. solani, com percentual de sobrevivência elevado. Em outros trabalhos, foi classificado como uma espécie de resistência moderada a forte, em áreas com histórico de F. solani (SILVA et al., 2013; PREISIGKE et al., 2015b), apesar de ter sido considerado suscetível, quando plantas de diferentes idades foram inoculadas com F. solani, via sistema radicular, em casa de vegetação (FISCHER et al., 2005b).
As plantas de $P$. foetida mostraram-se resistentes ao F. solani, com apenas um registro de mortalidade aos 140 DAT. Respostas positivas também foram registradas em trabalho realizado comparando cultivares de maracujazeiro amarelo, pé franco e enxertado em $P$. foetida, em uma área com histórico de $F$. oxysporum f. sp. passiflorae na região de Mossoró - RN, onde se verificaram 100\% de sobrevivência das plantas enxertadas (SILVA et al., 2017). Resultado semelhante 
foi observado em trabalho que avaliou o percentual de sobrevivência das plantas e as classificou de acordo com uma escala de avaliação da doença, em que a variabilidade intra e interespecífica da resistência contra F. solani foi confirmada, sendo $P$. foetida avaliado como altamente resistente (PREISIGKE et al., 2015a).

Esses resultados sugerem que $O P$. foetida é uma espécie promissora para o uso como porta-enxerto em regiões de solos com histórico de Fusarium spp., assim como para o melhoramento genético (SANTOS et al., 2011; PREISIGKE et al., 2015a; SILVA et al., 2017), destacando-se como uma alternativa tecnológica aos passicultores. No entanto, respostas divergentes foram verificadas por Roncatto et al. (2004), que descrevem essa espécie silvestre como sendo suscetível à morte precoce, ao avaliarem diferentes espécies de maracujazeiro em uma área com agentes fitopatogênicos desconhecidos, implantada na UNESP campus Jaboticabal.

Diante do exposto, fazem-se necessárias mais pesquisas sobre a sua adaptação sob diferentes condições climáticas em locais com histórico da podridão do colo e raiz, para que seja verificada a sua estabilidade ante o patógeno, uma vez que a interação da planta com o fungo pode ser influenciada por diversos fatores edafoclimáticos, como observado na interação significativa entre as espécies e o manejo de exposição parcial das raízes.

O maior percentual de sobrevivência das plantas de $P$. edulis, quando submetidas à exposição parcial do sistema radicular, sugere que medidas preventivas, que possam melhorar a aeração do colo e raízes e reduzir o atrito dessas estruturas com o solo, podem propiciar uma menor severidade da doença. As interações do F. solani e o sistema radicular das plantas estão intimamente ligadas às condições do ambiente rizosférico, uma vez que solos pesados e mal drenados favorecem 0 desenvolvimento da doença (FISCHER et al., 2008). 
Alguns autores descrevem maior patogenicidade e agressividade dos fungos após alterações na aeração da região radicular, assim como observado na cultura da ervilha, em que se verificou rápido crescimento micelial do F. solanif. sp. pisi em condições anaeróbicas, um incremento de 500\% no crescimento e aumento de $400 \%$ na podridão radicular de ervilha inoculadas (SMUCKER e ERICKSON, 1987), assim como foi observada maior severidade da podridão de raízes do feijoeiro, causada por Fusarium solanif. sp. phaseoli, em condições de atmosfera com deficiência de $\mathrm{O}_{2}$ (ALLMARAS et al., 1988).

Diante do exposto, faz-se necessária a utilização de ações integradas que visem a aumentar a supressividade do solo ao F. solani, priorizando medidas profiláticas que busquem melhorar as condições ambientais para desenvolvimento da cultura, tais como a melhoria da aeração do sistema radicular e colo da planta e a utilização de microrganismos promotores do crescimento vegetal e antagonistas a fitopatógenos.

\section{CONCLUSÕES}

A exposição parcial do sistema radicular reduz o percentual de plantas mortas por F. solani, aos 140 dias após o transplantio.

O Passiflora foetida apresenta, independente do manejo, alta resistência ao F. solani.

\section{AGRADECIMENTOS}

À Universidade Estadual do Sudoeste da Bahia campus Vitória da Conquista e ao Instituto Federal de Educação, Ciência e Tecnologia Baiano campus Guanambi, pelos insumos disponibilizados e à Fundação de Amparo à Pesquisa do Estado da Bahia, pela concessão da bolsa.

\section{REFERÊNCIAS}

ALLMARAS, R. R.; KRAFT, J. M. E MILLER, D. E. Effects of soil compaction and incorporated crop residue on root health. Annual Review of Phytopathology, v.26, p. 219-243, 1988. BOMFIM, M. P.; SÃO JOSÉ, A.R.; 
REBOUÇAS, T. N. H.; ALMEIDA, S. S.; SOUZA, I. V. B.; DIAS, N. O. Avaliação antagônica in vitro $\mathrm{e}$ in vivo de Trichoderma spp. a Rhizopus stolonifer em maracujazeiro amarelo. Summa Phytopathologica, Botucatu, v.36, p. 61-67, 2010.

BORGES, A. L.; SOUZA, L. D. Recomendações de calagem e adubação para maracujazeiro. Embrapa Mandioca e Fruticultura: Comunicado Técnico, 141, Cruz das Almas: Embrapa Mandioca e Fruticultura, 2010, 4 p.

BRASIL. Ministério das Minas Energia. Secretaria Geral. Projeto RAMBRASIL, Folha SD 23 Brasília; geologia, geomorfologia, pedologia, vegetação e uso potencial da terra. Rio de Janeiro, 1982, v.29, 660 p.

BUENO, C. J.; FISCHER, I. H.; PARISI, M. C. M. FURTADO, E. L. Comportamento do maracujazeiro amarelo, variedade Afruvec, ante uma população de Fusarium solani, agente causal da podridão do colo e raiz. Arquivos do Instituto Biológico, São Paulo, v.77, n. 3, p. 533-537, 2010.

CARVALHO, J. A.; JESUS, J. G.; ARAUJO, K. L. SERAFIM, M. E.; GILIO, T. A. S.; NEVES, L. G. Passion Fruit (Passiflora spp.) species as sources of resistance to soil phytopathogens Fusarium solani and Fusarium oxysporum f. sp. Passiflorae complex. Revista Brasileira de Fruticultura, Jaboticabal, v.43, n. 1, (Versão digital), 2021. DOI: http://dx.doi.org/10.1590/010029452021427
CAVICHIOLI, J. C.; CORRÊA, L. S.; MARCHI, M. J.; FISCHER, I. $H$. Desenvolvimento, produtividade e sobrevivência de maracujazeiroamarelo enxertado e cultivado em área com histórico de morte prematura de plantas. Revista Brasileira de Fruticultura, v.33, n. 2, p. 567-574, 2011. FERREIRA, R. T.; VIANA, A. P.; SILVA, F. H. de L. e.; SANTOS, E. A.; SANTOS, J. O. Seleção recorrente intrapopulacional em maracujazeiro azedo via modelos mistos. Revista Brasileira de Fruticultura, Jaboticabal, v.38, p. 158-166, 2016.

FIGUEIREDO, F. R. A.; HAFLE, O. M.; RODRIGUES, M. H. B. S.; PEREIRA JÚNIOR, E. B.; DELFINO, F. I. Produtividade e qualidade dos frutos do maracujazeiro amarelo sob diferentes formas de condução de plantas. Agropecuária Científica do Semiárido, v.11, n.4, p. 23-32, 2015.

FISCHER, I. H.; REZENDE, J. A. M. Diseases of passion flower (Passiflora spp.). Pest Technology, v.2, p. 1-19, 2008.

FISCHER, I. H.; KIMATI, H.; REZENDE, J. A. M. Doenças do maracujazeiro (Passifora spp.). In: KIMATI, H.; AMORIM, L.; REZENDE, J. A. M.; BERGAMIN FILHO, A.; CAMARGO, L. E. A. (Ed.). Manual de fitopatologia: Doenças das plantas cultivadas. v.2, 4.ed. São Paulo: Agronômica Ceres, 2005a, p.467-474.

FISCHER, I. H.; LOURENÇO, S. A.; MARTINS, M. C.; KIMATI, H.; 
AMORIM, L. Seleção de plantas resistentes e de fungicidas para $O$ controle

da podridão do colo e raiz do maracujazeiro causada por Nectria hematococca. Fitopatologia Brasileira, v.30, n.3, p. 250-258, 2005b.

JESUS, O. N.; FALEIRO, F. G.; JUNQUEIRA, N. T. V.; GIRARDI, E. A.; ROSA, R. C. C.; PETRY, H. B. Cultivares comerciais de maracujá-azedo (Passiflora edulis Sims) no Brasil. In: JUNGHANS, T. G.; JESUS, O. N. de. Maracujá do cultivo à comercialização, Brasília - DF: EMBRAPA, 2017, p. 3958.

JESUS, O. N. de; MARTINS, C.A.D.; OLIVEIRA, E. J.; SOARES, T. L.; FALEIRO, F.G.; BERNACCI, L.C.; MACHADO, C. de F.; GIRARDI, E. A.; ARAÚJO, F.P. de; PEREIRA, T.N.S.; VIANA, A.P.; JUNQUEIRA, N.T.V. Descritores morfoagronômicos ilustrados para Passiflora spp., Embrapa, Brasília, DF., 2016. 122 p.

KUDO, A. S.; PEIXOTO, J. R.; JUNQUEIRA, N. T. V; BLUM, L. E. B. Suscetibilidade de genótipos de maracujazeiro-azedo à septoriose em casa de vegetação. Revista Brasileira de Fruticultura, Jaboticabal, v. 34, n. 1, p. 200-205, 2012.

LARANJEIRA, F. F.; LIMA, A. A.; COSTA, M. M.; PFENNING, L. Progresso da fusariose do maracujá em portaenxertos do gênero Passiflora.

Fitopatologia Brasileira, Brasília, v.30, 2005, 146p.
MARCHIONATTO, J. B. Directivas en la lucha contra las enfermidades de las plantas. Revista Argentina de Agronomia, v.16, p. 29-32, 1949.

MAPA - Ministério da Agricultura Pecuária e Abastecimento. Base de dados AGROFIT. 2018. Disponível em: http://agrofit.agricultura.gov.br/agrofit _cons/principal_agrofit_cons. Acesso em: 04 jan. 2018.

MELETTI, L. M. M. Avanços na cultura do maracujá no Brasil. Revista Brasileira de Fruticultura, Jaboticabal, vol.33, n. spe1, p. 83-91, 2011.

PREISIGKE, S. C.; SILVA, L. P.; SERAFIM, M. E.; BRUCKNER, C. H.; ARAÚJO, K. L.; NEVES, L. G. Seleção precoce de espécies de Passiflora resistente a fusariose. Summa Phytopathologica, v.43, n. 4, p. 321-325, 2017.

PREISIGKE, S. C.; VIAN, M. F.; ROSSI, A. A. B.; SERAFIM, M. E.; BARELLI, M. A. A.; LUZ, P. B.; ARAÚJO, K. L.; NEVES, L. G. Genetic variability of 'Passiflora' spp. against collar rot disease, Autralian Journal of Crop Science, v.9, n. 1, 2015a. Disponível em: https://search.informit.com.au/docum entSummary; dn=915279997314246;res $=$ IELHS. Acesso em: 28 de junho de 2019.

PREISIGKE, S. C.; NEVES, L. G.; ARAÚJO, K. L.; BARBOSA, N. R.; SERAFIM, M. E.; KRAUSE, W. Multivariate analysis for the detection of Passiflora species resistant to collar rot. Bioscience Journal, Uberlândia, v.31, n. 6, p. 17001707, 2015b. 
RONCATTO, G.; OLIVEIRA, J.C.; RUGGIERO, C.; NOGUEIRA FILHO, G.C.; CENTURION, M.A.P.C.; FERREIRA, F.R. Comportamento de maracujazeiros (Passiflora spp.) quanto à morte prematura. Revista Brasileira de Fruticultura, Jaboticabal, v.26, n. 3, p. 552-554, 2004.

SÃO JOSÉ, A. R.; BOMFIM, M. P.; HOJO, R. H.; ANGEL, D. N.; PIRES, M. de M. Doenças do maracujazeiro. In: PIRES, M. M.; SÃO JOSÉ, A. R.; CONCEIÇÃO, A. O. Maracujá: avanços tecnológicos e sustentabilidade Ilhéus: Editus, 2011, p. 115-146.

SANTOS FILHO, H. P.; BARBOSA, F. F. L.; HADDAD, F. Doenças do maracujazeiro causadas por fungos, oomicetos e bactérias. In: JUNGHANS, T. G.; JESUS, O. N. de. Maracujá do cultivo à comercialização, Brasília DF: EMBRAPA, 2017, p.39-58.

SANTOS, E. A.; SOUZA, M. M.; VIANA, A. P.; ALMEIDA, A. A. F.; FREITAS, J. C. O.; LAWINSCKY, P. R. Multivariate analysis of morphological characteristics of two species of passion flower with ornamental potential and of hybrids between them. Genetics and Molecular Research, Ribeirão Preto, v.10, n. 4, p. 2457-2471, 2011.

SILVA, R. M.; AMBRÓSIO, M. M. Q.; AGUIAR, A. V. M.; FALEIRO, F. G.; CARDOSO, A. M. S.; MENDONÇA, V. Reaction of passion fruit cultivars in areas with fusariosis. Summa Phytopathologica, v.43, n.2, p.98-102, 2017.
SILVA, A. N.; AZEVEDO, G. B.; ROCHA SOBRINHO, G. G.; NOVAES, Q. S. Efeito de produtos químicos e de Trichoderma spp. no controle de Fusarium solani do maracujazeiro. Interciencia, v.39, n. 6, p. 398-403, 2014.

SILVA, A. S.; OLIVEIRA, E. J.; HADDAD, F.; LARANJEIRA, F. F.; JESUS, O. N.; OLIVEIRA, S. A. S.; COSTA, M. A. P. C.; FREITAS, J. P. X. Identification of passion fruitn genotypes resistant to Fusarium oxysporum f. sp. passiflorae. Tropical Plant Pathology, Brasilia, v.38, n. 3, p. 236-242, 2013.

SMUCKER, A. J. M., ERICKSON, A. E. Anaerobic stimulation of root exudates and disease of peas. Plant Soil, v.99, p. 423-433, 1987. 